\title{
Does a relativistic metric generalization of Newtonian gravity exist in $2+1$ dimensions?
}

\author{
J.L. Alonso, J.L. Cortés, and V. Laliena \\ Departamento de Física Teórica, Universidad de Zaragoza, \\ C. Pedro Cerbuna 12, E-50009 Zaragoza (Spain)
}

(Dated: October 23, 2002)

\begin{abstract}
It is shown that, contrary to previous claims, a scalar tensor theory of Brans-Dicke type provides a relativistic generalization of Newtonian gravity in $2+1$ dimensions. The theory is metric and test particles follow the space-time geodesics. The static isotropic solution is studied in vacuum and in regions filled with an incompressible perfect fluid. It is shown that the solutions can be consistently matched at the interface matter-vacuum, and that the Newtonian behavior is recovered in the weak field regime.
\end{abstract}

\section{INTRODUCTION}

Newtonian gravity, a theory of gravitational phenomena which is invariant under Galilean transformations and, therefore, valid only in the low energy (weak fields and slow motions) regime, must be generalized to a RelRelativity (EGR) is a good candidate for RTG in $3+1$ dimensions, but other possibilities, as Brans-Dicke theory (BDT) [1], have been proposed. Indeed, it is believed by many that a quantum theory of gravitation, which seems unavoidable if we want to deal with gravitational phenomena at the Planck scale, must contain something more than ERG [2].

In $2+1$ dimensions, EGR is not a RTG. The RiemannChristoffel tensor is uniquely determined by the Ricci tensor, which vanishes outside the sources. Hence, spacetime is flat in regions devoid of matter, the geodesics are straight lines and test particles do not feel any gravitational field [3]. A proper RTG in $2+1$ dimensions needs some additional ingredient besides the metric tensor of EGR [4]. A minimal candidate for RTG is a scalar tensor theory of Brans-Dicke type. It has been claimed that even theories of this sort, which are much richer than EGR, do not describe Newtonian gravity in the low energy limit [5]. We will show in this paper that BDT in $2+1$ dimensions reproduces Newtonian gravity when the low energy regimen is consistently analysed. The additional ingredient that seems necessary to construct a quantum theory of gravitation in $3+1$ dimensions appears at an earlier stage in lower dimensions.

The construction of a RTG in dimensions lower than $3+1$ is interesting because it may allow to study phenomena characteristic of gravity, which have $3+1$ dimensional analogues, such as gravitational instabilities and black holes, in a simplified context [6, 7].

The paper is organized as follow. In section II the equations of scalar-tensor theories in $D$-dimensional spacetime are derived, the particular case of BDT is identiativistic Theory of Gravitation (RTG). Einstein General

fied, and the peculiarities of $D=3$ and $D=2$ are discussed. Section III is devoted to the study of the weak field limit and in section IV the fields corresponding to static isotropic extended sources in $D=3$ are analysed. The solutions inside and outside the source can be consistently matched and reproduce the Newton gravitational field in the weak field limit. Finally, section V contains some concluding remarks.

\section{SCALAR-TENSOR THEORIES IN D-DIMENSIONS}

The minimal modification of EGR introduces, besides the metric tensor, a scalar field associated to the gravitational interaction. The most natural way to interpret it is noting that it sets the strength of the gravitational coupling at each space-time point, which is thus determined by the mass distribution of the universe. It has the advantage that it incorporates Mach principle better than EGR. The fact that the scalar field is related to the gravitational interaction strength implies that its coupling to the metric tensor cannot be minimal: it must be of the type scalar-tensor interaction. The action for the gravitational sector in such kind of theories in $D$-dimensional space-time is

$$
\mathcal{S}_{g}=-\int d^{D} x \sqrt{g}\left[\phi R+W(\phi) g^{\mu \nu} \phi_{; \mu} \phi_{; \nu}\right]
$$

where $g_{\mu \nu}$ is the metric tensor, whose signature is taken $(-,+, \ldots,+), g=-\operatorname{det} g_{\mu \nu}, R$ is the scalar of curvature, and $\phi$ is the scalar field that acts as the inverse gravitational coupling at each space-time point. The action contains an unspecified function of $\phi, W$. Different choices of such function give different scalar tensor theories.

The coupling of the matter to gravity depends only on the metric tensor in a covariant way, and does not depend of the scalar field. Hence, the variation of the matter action, $\mathcal{S}_{m}$, under variations of the metric, $\delta g_{\mu \nu}$, *Electronic address: buj@gteorico.unizar.es,cortes@leo.unizar.es,laliena@ansbeunividtex as $\delta \mathcal{S}_{m}=1 / 2 \int d^{D} x \sqrt{g} T^{\mu \nu} \delta g_{\mu \nu}$, and gen- 
eral covariance implies a continuity equation for $T^{\mu \nu}$ :

$$
T_{; \nu}^{\mu \nu}=0
$$

that describes the exchange of energy between matter and gravity. Furthermore, this equation ensures that test particles move along the geodesics of space-time associated to the metric $g_{\mu \nu}$ and thus the equivalence principle is preserved. In this sense, scalar-tensor theories are metric theories of gravitation.

The equations for the metric tensor and the scalar field that follow from the action $\mathcal{S}=\mathcal{S}_{g}+\mathcal{S}_{m}$ are

$$
\begin{aligned}
\phi\left[R_{\mu \nu}-\frac{1}{2} g_{\mu \nu} R\right]+\left(g_{\mu}^{\rho} g_{\nu}^{\sigma}-g_{\mu \nu} g^{\rho \sigma}\right) \phi_{; \rho ; \sigma}+W(\phi)\left[g_{\mu}^{\rho} g_{\nu}^{\sigma}-\frac{1}{2} g_{\mu \nu} g^{\rho \sigma}\right] \phi_{; \rho} \phi_{; \sigma} & =-\frac{1}{2} T_{\mu \nu} \\
R-g^{\rho \sigma}\left[2 W(\phi) \phi_{; \rho ; \sigma}+W^{\prime}(\phi) \phi_{; \rho} \phi_{; \sigma}\right] & =0
\end{aligned}
$$

where the prime means derivation with respect to $\phi$.

For $D \neq 2$, Eq. (3a) allows to express $R$ as a function of $\phi$ and its covariant derivatives and the trace of the energy-momentum tensor, $T=g_{\mu \nu} T^{\mu \nu}$. Thus, Eqs. (3) can be written in the following form:

$$
\begin{aligned}
& \phi R_{\mu \nu}=-\left[\frac{1}{D-2} g_{\mu \nu} g^{\rho \sigma}+g_{\mu}^{\rho} g_{\nu}^{\sigma}\right] \phi_{; \rho ; \sigma}-W(\phi) \phi_{; \mu} \phi_{; \nu}-\frac{1}{2}\left[T_{\mu \nu}-\frac{1}{D-2} g_{\mu \nu} T\right] \\
& 2\left[\frac{D-1}{(D-2) \phi}+W(\phi)\right] g^{\rho \sigma} \phi_{; \rho ; \sigma}+\left[\frac{W(\phi)}{\phi}+W^{\prime}(\phi)\right] g^{\rho \sigma} \phi_{; \rho} \phi_{; \sigma}-\frac{1}{(D-2) \phi} T=0 .
\end{aligned}
$$

The above equations are particularly simple with the choice $W(\phi)=\omega / \phi$, where $\omega$ is a constant. In this case we get the field equations of Brans-Dicke theory [1]:

$$
\begin{aligned}
& g^{\rho \sigma} \phi_{; \rho ; \sigma}=\frac{\lambda}{2(1+\omega)} T \\
& \phi R_{\mu \nu}=-\left[\phi_{; \mu ; \nu}+\frac{\omega}{\phi} \phi_{; \mu} \phi_{; \nu}\right]-\frac{1}{2}\left[T_{\mu \nu}-\lambda g_{\mu \nu} T\right]
\end{aligned}
$$

where $\lambda$ is a function of $\omega$ and $D$ :

$$
\lambda=\frac{1+\omega}{(D-1)+\omega(D-2)} .
$$

Introducing the parametrisation $\phi=\frac{1}{\mathcal{G}} \mathrm{e}^{\xi}$ (then $\phi_{; \mu}=$ $\phi \xi_{; \mu}$ and $\left.\phi_{; \mu ; \nu}=\phi\left(\xi_{; \mu ; \nu}+\xi_{; \mu} \xi_{; \nu}\right)\right)$, where $\xi$ is dimensionless and the constant $\mathcal{G}$ appears for dimensional reasons, the equations take the form

$$
\begin{aligned}
& g^{\rho \sigma}\left(\xi_{; \rho ; \sigma}+\xi_{; \rho} \xi_{; \sigma}\right)=\frac{\mathcal{G}}{2} \mathrm{e}^{-\xi} \frac{\lambda}{1+\omega} T \\
& R_{\mu \nu}=-\xi_{; \mu ; \nu}-(1+\omega) \xi_{; \mu} \xi_{; \nu}-\frac{\mathcal{G}}{2} \mathrm{e}^{-\xi}\left[T_{\mu \nu}-\lambda g_{\mu \nu} T\right]
\end{aligned}
$$

Such parametrisation assumes only that the scalar field has a definite sign and, as we shall see, is appropiate to analyse the existence of the Newtonian limit.

The case $D=2$ is especial, since $R_{\mu \nu}=\frac{1}{2} g_{\mu \nu} R$, and the equations read

$$
\begin{gathered}
g^{\rho \sigma}\left(\xi_{; \rho ; \sigma}+\xi_{; \rho} \xi_{; \sigma}\right)=\frac{\mathcal{G}}{2} e^{-\xi} T, \\
R=\omega\left(\mathcal{G} e^{-\xi} T-g^{\rho \sigma} \xi_{; \rho} \xi_{; \sigma}\right) .
\end{gathered}
$$

\section{PERTURBATIVE SOLUTION IN D-DIMENSIONS}

If the energy-momentum tensor of matter, $T^{\mu \nu}$, vanishes, the system of equations (7) have the vacuum solution $g_{\mu \nu}=\eta_{\mu \nu}$ and $\xi=0$, where $\eta_{\mu \nu}$ is the Minkowski metric tensor. This solution fixes the arbitrariness in the coordinate system and definition of $\mathcal{G}$. For a weak $T_{\mu \nu}$ the solution of Eqs. (7) can be obtained perturbatively as a series in powers of $\mathcal{G}$ :

$$
\begin{aligned}
\xi(x) & =\sum_{n=1}^{\infty} \mathcal{G}^{n} \xi^{(n)}(x) \\
g_{\mu \nu}(x) & =\eta_{\mu \nu}+\sum_{n=1}^{\infty} \mathcal{G}^{n} g_{\mu \nu}^{(n)}(x) \\
T_{\mu \nu}(x) & =T_{\mu \nu}^{(0)}(x)+\sum_{n=1}^{\infty} \mathcal{G}^{n} T_{\mu \nu}^{(n)}(x),
\end{aligned}
$$

where $T_{\mu \nu}^{(0)}$ is the energy-momentum tensor of matter in the absence of gravitation. Imposing Eqs. (7) and the continuity equation (2) order by order in $\mathcal{G}$ leads to a set 
of linear equations for $\xi^{(n)}, g_{\mu \nu}^{(n)}$, and $T_{\mu \nu}^{(n)}$. To first order in $\mathcal{G}$, Eqs. (7) yield

$$
\begin{aligned}
& \eta^{\rho \sigma} \frac{\partial^{2} \xi^{(1)}}{\partial x^{\rho} \partial x^{\sigma}}=\frac{\lambda}{2(1+\omega)} T^{(0)}, \\
& R_{\mu \nu}^{(1)}=-\frac{\partial^{2} \xi^{(1)}}{\partial x^{\mu} \partial x^{\nu}}-\frac{1}{2}\left[T_{\mu \nu}^{(0)}-\lambda \eta_{\mu \nu} T^{(0)}\right] .
\end{aligned}
$$

For a static field produced by non-relativistic matter $\left(T_{\mu \nu}^{(0)}=0\right.$ for $(\mu, \nu) \neq(0,0)$ and $T_{00}^{(0)}=\rho$, where $\rho$ is the density of matter), $R_{00}^{(1)}=\frac{1}{2} \nabla^{2} g_{00}^{(1)}$. In this case, for $D>2$ we have:

$$
\nabla^{2} g_{00}^{(1)}=-(1-\lambda) \rho,
$$

and for $D=2$ :

$$
\nabla^{2} g_{00}^{(1)}=-\frac{\omega}{4} \rho .
$$

The Newtonian potential is identified from the geodesic equation as $V_{\mathrm{N}}=-\frac{\mathcal{G}}{2} g_{00}^{(1)}$. Remembering that the Newtonian potential in $D-1$ spatial dimensions verifies the Poisson equation

$$
\nabla^{2} V_{\mathrm{N}}=S_{D-2} G_{\mathrm{N}} \rho
$$

where $S_{n}$ is the area of the n-dimensional unit sphere [8] and $G_{\mathrm{N}}$ the Newton constant, we have for $D>2$

$$
G_{\mathrm{N}}=\frac{\mathcal{G}}{2 S_{D-2}}(1-\lambda)
$$

and for $D=2$

$$
G_{\mathrm{N}}=\frac{\mathcal{G} \omega}{4} .
$$

As expected, we see that $G_{\mathrm{N}}$ vanishes as $\omega \rightarrow \infty$ in $D=$ 3 , and it tends to $\mathcal{G} / 16 \pi$ in $D=4$.

Note that the Newtonian limit of the scalar-tensor with a coupling as given in Eqs. (14) and (15) is compatible with a variable effective gravitational coupling proportional to the reciprocal of the scalar field, $G_{\mathrm{eff}}=\frac{1-\lambda}{2 S_{D-2}} \frac{1}{\phi}$. In fact, $G_{\mathrm{N}}$ is just the first order term in an expansion of $G_{\text {eff }}$ in powers of $\mathcal{G}$.

\section{FIELD OF STATIC ISOTROPIC SOURCES IN $\mathrm{D}=3$}

The perturbative expansion is rather formal and it is interesting to see that it is consistent in the most important case of static and isotropic sources. To this end, let us compute the field produced by a static and isotropic source in regions devoid of sources in $D=3$. In a suitable reference system, the metric can be written in the standard form:

$$
d \tau^{2}=\mathrm{e}^{2 \beta} d t^{2}-\mathrm{e}^{2 \alpha} d r^{2}-r^{2} d \varphi^{2},
$$

where $\alpha$ and $\beta$ are functions of $r$ alone. In vacuum $\left(T_{\mu \nu}=\right.$ 0) Eqs. (7) yield

$$
\begin{aligned}
\xi & =\xi_{0}+C_{\xi} \ln \left(r / r_{0}\right), \\
\alpha & =\alpha_{0}+C_{\alpha} \ln \left(r / r_{0}\right), \\
\beta & =\beta_{0}+C_{\beta} \ln \left(r / r_{0}\right),
\end{aligned}
$$

where $r_{0}$ is some arbitrary length scale, $\xi_{0}, \alpha_{0}$, and $\beta_{0}$ and $C_{\xi}$ are constants, and

$$
\begin{aligned}
C_{\alpha} & =\frac{1}{2} \frac{(\omega+2) C_{\xi}^{2}}{1+C_{\xi}}, \\
C_{\beta} & =\frac{1}{2} \frac{\omega C_{\xi}^{2}-2 C_{\xi}}{1+C_{\xi}} .
\end{aligned}
$$

The fields are singular at the origin and must be produced by some source extended about (or concentrated at) the origin. The constant $C_{\xi}$ is determined by the source as follows: multiplying Eq. (5a) by $\sqrt{g}$ and integrating in $d r d \varphi$, and assuming that a static and isotropic source is confined within a finite spatial region, we get

$$
C_{\xi}=-\exp \left(\alpha_{0}-\beta_{0}-\xi_{0}\right) \frac{\mathcal{G}}{4 \pi(\omega+2)} M,
$$

where the mass, $M$, is defined as $M=\int d r d \varphi \sqrt{g} T$.

In the weak field regime $\mathcal{G} M$ is small. Eqs. (9a)-(9b) imply that $\xi_{0}, \alpha_{0}$, and $\beta_{0}$ are proportional to $\mathcal{G}$. Then to first order in $\mathcal{G}$ we have

$$
\mathrm{e}^{2 \beta} \approx 1+2 \frac{\mathcal{G}}{4 \pi(2+\omega)} M \ln \frac{r}{r_{0}} .
$$

A comparison with the Newtonian solution, $V_{\mathrm{N}}=$ $G_{\mathrm{N}} M \ln \left(r / r_{0}\right)$, gives the Newton constant $G_{\mathrm{N}}=$ $\mathcal{G} /[4 \pi(2+\omega)]$, the same obtained from field equations in presence of matter in the weak field regime.

Eqs. (7) do not admit solutions with a static point mass as source [5]. Indeed, they do not admit static and isotropic solutions with concentrated sources (energymomentum tensor that contains Dirac deltas). The reason is that it is not possible to find solutions $\alpha, \beta$, and $\xi$ of the sourceless equations with singularities cancelling the source singularities in the field equations. We are thus lead to consider extended sources. This is interesting because doubts have been cast on the existence of static and isotropic solutions of Eqs. (7) even with extended sources [5]. To investigate this in depth, let us consider as source 
an incompressible fluid of density $\rho$ confined on a disk of radius $r_{\mathrm{M}}$. The corresponding energy-momentum tensor is

$$
T_{\mu \nu}=p g_{\mu \nu}+(\rho+p) U_{\mu} U_{\nu},
$$

where $p$ is the pressure and $U_{\mu}$ the four velocity, which verifies $g^{\mu \nu} U_{\mu} U_{\nu}=-1$. For a static fluid we have

$$
T_{t t}=\mathrm{e}^{2 \beta} \rho, \quad T_{r r}=\mathrm{e}^{2 \alpha} p, \quad T_{\varphi \varphi}=r^{2} p,
$$

and the remaining components vanish. Covariant conservation of $T_{\mu \nu}$ implies the equation of hydrostatic equilibrium [9]:

$$
\beta^{\prime}=-\frac{p^{\prime}}{p+\rho}
$$

where the prime indicates derivation respect to $r$. Using the dimensionless variable $\hat{r}=r / r_{\mathrm{M}}$ and $\bar{p}=p / \rho$, and the dimensionless parameter $\kappa=\mathcal{G} r_{\mathrm{M}}^{2} \rho /[2(2+\omega)]$, Eqs. (7) read:

$$
\begin{aligned}
\xi^{\prime \prime}+\frac{1}{\hat{r}} \xi^{\prime}+\xi^{\prime}\left(\beta^{\prime}+\xi^{\prime}-\alpha^{\prime}\right) & =-\kappa(1-2 \bar{p}) \mathrm{e}^{2 \alpha-\xi}, \\
\beta^{\prime \prime}+\frac{1}{\hat{r}} \beta^{\prime}+\beta^{\prime}\left(\beta^{\prime}+\xi^{\prime}-\alpha^{\prime}\right) & =\kappa[1+2(1+\omega) \bar{p}] \mathrm{e}^{2 \alpha-\xi}, \\
\beta^{\prime \prime}-\frac{1}{\hat{r}} \alpha^{\prime}+\xi^{\prime \prime}+\beta^{\prime 2}+(1+\omega) \xi^{\prime 2}-\alpha^{\prime}\left(\beta^{\prime}+\xi^{\prime}\right) & =-\kappa[1+\omega-\omega \bar{p}] \mathrm{e}^{2 \alpha-\xi}, \\
\frac{1}{\hat{r}}\left(\beta^{\prime}+\xi^{\prime}-\alpha^{\prime}\right) & =-\kappa[1+\omega-\omega \bar{p}] \mathrm{e}^{2 \alpha-\xi},
\end{aligned}
$$

where now the primes stand for derivation respect to $\hat{r}$.

Notice that there is no static and isotropic solution for dust $(p=0)$, since then $\beta^{\prime}=0$ and this forces $\kappa=0$, i.e., $\rho=0$.

With nonzero $\kappa$, the system of Eqs. (24), supplemented with (23), is very complicated. For $\kappa=0$ there is a trivial solution : $\xi=\alpha=\beta=\bar{p}=0$. For $\kappa \neq 0$ the solution can be found iteratively, by an expansion in powers of $\kappa$ :

$$
\begin{aligned}
\xi & =\sum_{i=1}^{\infty} \kappa^{i} \xi_{i}, & \alpha & =\sum_{i=1}^{\infty} \kappa^{i} \alpha_{i}, \\
\beta & =\sum_{i=1}^{\infty} \kappa^{i} \beta_{i}, & \bar{p} & =\sum_{i=1}^{\infty} \kappa^{i} \bar{p}_{i} .
\end{aligned}
$$

At order $\kappa^{i}$ the equations for $\xi_{i}, \alpha_{i}$, and $\beta_{i}$ are linear and very easy to solve. The solutions, however, become more and more cumbersome as the order increases. Table I displays the solution up to order $\kappa^{4}$, with the boundary conditions discussed below. With the aid of programs of symbolic calculus one can easily find the solution up to very large orders.

Boundary conditions are fixed by requiring that $\alpha, \beta$, and $\xi$ be regular at the origin. This eliminates the singular solutions, which contain $\ln \hat{r}$, and fixes two integration constants. The ambiguity in the choice of a reference system and a scale for the gravitational interaction $(\mathcal{G}$ has been arbitrarily chosen) is reflected in the presence of three more integration constants. They can be fixed, for instance, by requiring that at the origin the coordinate system be locally inertial and $\xi$ vanish. In addition, we require that the pressure vanishes at $\hat{r}=1$. In this way, the solution is completely determined.

Let us analyze the matching with the vacuum solution
(17) for $\hat{r}>1$. It is convenient to choose as length scale $r_{0}=r_{\mathrm{M}}$. The boundary conditions at $\hat{r}=1$ are the continuity of $\alpha, \beta, \xi, \beta^{\prime}$, and $\xi^{\prime}$. Since $T_{\mu \nu}$ has a jump at $\hat{r}=1$, it follows that $\beta^{\prime \prime}, \xi^{\prime \prime}$, and $\alpha^{\prime}$ must also have a jump. The constants $\alpha_{0}, \beta_{0}$, and $\xi_{0}$ must be chosen to guarantee the continuity of $\alpha, \beta$, and $\xi$. For the derivatives we have:

$$
\begin{aligned}
& \sum_{i=1}^{\infty} \kappa^{i} \xi_{i}^{\prime}(\hat{r}=1)=C_{\xi}, \\
& \sum_{i=1}^{\infty} \kappa^{i} \beta_{i}^{\prime}(\hat{r}=1)=C_{\beta} .
\end{aligned}
$$

Although $C_{\xi}$ and $C_{\beta}$ are linked by Eq. (18b), the above two equations are simultaneously satisfied since Eqs. (24) guarantee that the following relation holds at the points where the pressure vanishes:

$$
2 \beta^{\prime}\left(\frac{1}{\hat{r}}+\xi^{\prime}\right)=\omega \xi^{\prime 2}-\frac{2}{\hat{r}} \xi^{\prime} .
$$

Thus, the condition that pressure vanishes at $\hat{r}=1$ is not only physically sound, but necessary to match with the vacuum solution.

The matching can be made order by order in $\kappa$. In such case we have

$$
C_{\xi}=\sum_{i=1}^{\infty} \kappa^{i} C_{\xi}^{(i)}
$$


As a check, it can be seen that Eq. (19) is verified.

The solution up to order $\kappa^{4}$ is displayed in Table I. The fields $\beta$ and $\alpha$ are positive and monotonically increasing order by order, and the preasure is positive and monotonically decreasing order by order, vanishing at the border of the mass distribution $(\hat{r}=1)$. This is in agreement with intuition, since a positive preasure is required to balance the gravitational attraction. Gravitational collapse is impossible since the fluid is incompressible.

Up to order $\kappa^{4}$, the limit $\omega \rightarrow \infty$ gives (recall that $\kappa \sim 1 / \omega) \xi=\beta=\bar{p}=C_{\xi}=0$ and

$$
\alpha=\frac{1}{2} \bar{\kappa} \hat{r}^{2}+\frac{1}{4} \bar{\kappa}^{2} \hat{r}^{4}+\frac{1}{6} \bar{\kappa}^{3} \hat{r}^{6}+\frac{1}{8} \bar{\kappa}^{4} \hat{r}^{8},
$$

where $\bar{\kappa}=\mathcal{G} r_{\mathrm{M}}^{2} \rho / 2$. On the other hand, we can take the limit $\omega \rightarrow \infty$ of Eqs. (24). This forces $\xi^{\prime}=0$ and then the solution with the boundary conditions previously described is $\xi=\beta=\bar{p}=0$ and

$$
\alpha=-\frac{1}{2} \ln \left(1-\bar{\kappa} \hat{r}^{2}\right) .
$$

We see that Eq. (29) is the Taylor expansion of the exact solution (30) around $\bar{\kappa} \hat{r}^{2}=0$. Since $\hat{r} \leq 1$, the expansion will converge for $\bar{\kappa}<1$. For finite $\omega$, the convergence radius of the expansion in powers of $\kappa$ is an open question.

We can understand that the preasure is zero in the $\omega \rightarrow \infty$ limit by recalling that EGR in $2+1$ dimensions produces no force between separated sources. If we represent a continuous mass distribution as a continuum limit of localized sources, we understand that the preasure necessary to balance gravity is zero.

The field of a static isotropic source in Brans-Dicke theory in $D=2$ has been obtained in [7]. It can be seen that the Newton constant obtained from it agrees with Eq. (15).

\section{FINAL COMMENTS}

We have seen that BDT provides a RTG in D dimensions. In dimension lower than four, EGR is not a RTG, and the new ingredient present in BDT, the scalar field, is essential to have a Newtonian regime at low energies. The dynamics induced by the new field contains all features that one expects in a theory of gravitation, so that it can be used to check phenomena such as gravitational instabilities and black holes in the simplified context of lower dimensional worlds.

In four dimensions, it is believed that a consistent quantum theory of gravitation must contain, besides the metric tensor, additional fields like the dilaton. It is remarkable that in lower dimensions the necessity for such new ingredients appear already at an earlier stage.

This work has been partially supported by MCYT (Spain), grants FPA2000-1252 and FPA2001-1813. V.L. has been supported by the Ramón y Cajal program.
[1] C. Brans and R.H. Dicke, Phys. Rev. 164, 925 (1961).

[2] Scalar fields associated to the metric are present in superstring theories: B. Green, J.M. Schwarz and E. Witten, Superstring Theory (Cambridge University Press, Cambridge, England, 1987).

[3] S. Deser, R. Jackiw, and G. 't Hooft, Ann. Phys. (N.Y.) 152, 220 (1984).

[4] There is a metric theory in $2+1$ dimensions in which the curvature tensor vanishes and gravity is due to torsion (teleparallel theory). It gives the correct Newtonian limit and is, therefore, a RTG. It has been developed by T. Kawai, Phys. Rev. D48, 5668 (1993).

[5] J.D. Barrow, A.B. Burd, and D. Lancaster, Class. Quantum Grav. 3, 551 (1986).(Eq. (2.9) of this Ref. has a typo: $\omega$ must read $\omega / \Phi$.

[6] N.J. Cornish and N.E. Frankel, Phys. Rev. D43, 2555 (1991); ibid 47, 714 (1993). M. Bañados, C. Teitelboim, and J. Zanelli, Phys. Rev. Lett. 69, 1849 (1992). P.M. Sá and J.P. Lemos, Class. Quantum Grav. 13, 125 (1996). M. Cadoni, Phys. Rev. D54, 7378 (1996). J.P. Lemos and P.M. Sá, Class. Quantum Grav. 11, L11 (1994).

[7] J. P. Lemos and P.M. Sá, Phys. Rev. D49, 2897 (1994); Erratum, ibid 51, 5967 (1995).

[8] Eg. $S_{0}=2, S_{1}=2 \pi, S_{2}=4 \pi$, etc.

[9] S. Weinberg, Gravitation and Cosmology, Wiley, New York (1972). 


\begin{tabular}{|c|c|}
\hline$\xi_{1}$ & $-\frac{1}{4} \hat{r}^{2}$ \\
\hline$\xi_{2}$ & $\frac{1}{8} \hat{r}^{2}-\frac{1}{64}(9+6 \omega) \hat{r}^{4}$ \\
\hline$\xi_{3}$ & $\frac{1}{128}\left[(15+12 \omega) \hat{r}^{2}+(1+5 \omega) \hat{r}^{4}-\frac{1}{6}\left(40 \omega^{2}+114 \omega+71\right) \hat{r}^{6}\right]$ \\
\hline$\xi_{4}$ & $\begin{array}{l}\frac{1}{192}\left(26+38 \omega+15 \omega^{2}\right) \hat{r}^{2}+\frac{1}{2048}\left(31+71 \omega+60 \omega^{2}\right) \hat{r}^{4}+\frac{1}{1536}\left(32 \omega^{2}+29 \omega-27\right) \hat{r}^{6} \\
-\frac{1}{24576}\left(1710+4457 \omega+3508 \omega^{2}+840 \omega^{3}\right) \hat{r}^{8}\end{array}$ \\
\hline$\beta_{1}$ & $\frac{1}{4} \hat{r}^{2}$ \\
\hline$\beta_{2}$ & $\frac{1}{8}(1+\omega) \hat{r}^{2}+\frac{1}{64}(5+4 \omega) \hat{r}^{4}$ \\
\hline$\beta_{3}$ & $\frac{1}{128}\left[\left(12 \omega^{2}+27 \omega+15\right) \hat{r}^{2}+\left(4 \omega^{2}+7 \omega+7\right) \hat{r}^{4}+\frac{1}{6}\left(24 \omega^{2}+64 \omega+35\right) \hat{r}^{6}\right]$ \\
\hline$\beta_{4}$ & $\begin{array}{l}\frac{1}{192}\left(26+64 \omega+53 \omega^{2}+15 \omega^{3}\right) \hat{r}^{2}+\frac{1}{2048}\left(121+189 \omega+128 \omega^{2}+48 \omega^{3}\right) \hat{r}^{4} \\
+\frac{1}{1536}\left(63+109 \omega+76 \omega^{2}+24 \omega^{3}\right) \hat{r}^{6}+\frac{1}{24576}\left(802+2291 \omega+1932 \omega^{2}+480 \omega^{3}\right) \hat{r}^{8}\end{array}$ \\
\hline$\alpha_{1}$ & $\frac{1}{2}(1+\omega) \hat{r}^{2}$ \\
\hline$\alpha_{2}$ & $\frac{1}{4} \hat{r}^{2}+\frac{1}{32}\left(8 \omega^{2}+19 \omega+8\right) \hat{r}^{4}$ \\
\hline$\alpha_{3}$ & $\frac{1}{64}(12 \omega+15) \hat{r}^{2}+\frac{5}{32}(\omega+1) \hat{r}^{4}+\frac{1}{384}\left(64 \omega^{3}+238 \omega^{2}+251 \omega+73\right) \hat{r}^{6}$ \\
\hline$\alpha_{4}$ & $\begin{array}{l}\frac{1}{96}\left(15 \omega^{2}+38 \omega+26\right) \hat{r}^{2}+\frac{1}{512}\left(60 \omega^{2}+123 \omega+83\right) \hat{r}^{4}+\frac{1}{256}\left(35 \omega^{2}+84 \omega+39\right) \hat{r}^{6} \\
+\frac{1}{12288}\left(1536 \omega^{4}+7756 \omega^{3}+13232 \omega^{2}+8933 \omega+2002\right) \hat{r}^{8}\end{array}$ \\
\hline $\bar{p}_{1}$ & $\frac{1}{4}-\frac{1}{4} \hat{r}^{2}$ \\
\hline $\bar{p}_{2}$ & $\frac{1}{64}\left[12 \omega+15-(8 \omega+12) \hat{r}^{2}-(4 \omega+3) \hat{r}^{4}\right]$ \\
\hline $\bar{p}_{3}$ & $\frac{13}{48}+\frac{19}{48} \omega+\frac{5}{32} \omega^{2}-\frac{1}{256}\left(53+74 \omega+24 \omega^{2}\right) \hat{r}^{2}-\frac{1}{256}\left(9+10 \omega+8 \omega^{2}\right) \hat{r}^{4}-\frac{1}{384}\left(11+26 \omega+12 \omega^{2}\right) \hat{r}^{6}$ \\
\hline $\bar{p}_{4}$ & $\begin{array}{l}\frac{1}{24576}\left(8593+17719 \omega+12860 \omega^{2}+3360 \omega^{3}\right)-\frac{1}{768}\left(201+413 \omega+278 \omega^{2}+60 \omega^{3}\right) \hat{r}^{2} \\
-\frac{1}{4096}\left(159+218 \omega+208 \omega^{2}+96 \omega^{3}\right) \hat{r}^{4}-\frac{1}{1536}\left(44+93 \omega+64 \omega^{2}+24 \omega^{3}\right) \hat{r}^{6} \\
-\frac{1}{24576}\left(503+1707 \omega+1692 \omega^{2}+480 \omega^{3}\right) \hat{r}^{8}\end{array}$ \\
\hline$C_{\xi}^{(1)}$ & $-\frac{1}{2}$ \\
\hline$C_{\xi}^{(2)}$ & $-\frac{1}{16}(5+6 \omega)$ \\
\hline$C_{\xi}^{(3)}$ & $-\frac{1}{128}\left(37+70 \omega+40 \omega^{2}\right)$ \\
\hline$C_{\xi}^{(4)}$ & $-\frac{1}{3072}\left(1016+2467 \omega+2284 \omega^{2}+840 \omega^{3}\right)$ \\
\hline
\end{tabular}

TABLE I: Solution of Eqs. (24) up to order $\kappa^{4}$. The boundary conditions are described in section IV 